\title{
Motile Bacteria at Oil-Water Interfaces: Pseudomonas Aeruginosa
}

\author{
Jiayi Deng, Mehdi Molaei, Nicholas G. Chisholm, and Kathleen J. Stebe \\ Department of Chemical and Biomolecular Engineering, University of Pennsylvania, \\ Philadelphia \\ E-mail: kstebe@seas.upenn.edu
}

\section{Supporting Information}

\section{SI1. The bounded mean-squared angular displacement (MSAD)}

The MSAD of the bacteria in the absents of static noise follows a stretched exponential function [1] since the measured orientation of the cell body $\phi$ is bounded between 0 and $\pi / 2$.

$$
\left\langle\Delta \phi^{2}(\tau)\right\rangle_{b}=\left\langle\Delta \phi^{2}(\infty)\right\rangle_{b}\left[1-\exp \left(-\xi\left(2 D_{R}\right)^{\zeta}\right)\right]
$$

At large lag time, the probability distribution of $\phi$ is $P(\phi ; t \rightarrow \infty)=2 / \pi$. Thus,

$$
\left\langle\Delta \phi^{2}(\infty)\right\rangle_{b}=\frac{4}{\pi^{2}} \int_{0}^{\frac{\pi}{2}} d \phi_{2} \int_{0}^{\frac{\pi}{2}}\left(\phi_{1}-\phi_{2}\right)^{2} d \phi_{1}=\frac{\pi^{2}}{24}
$$

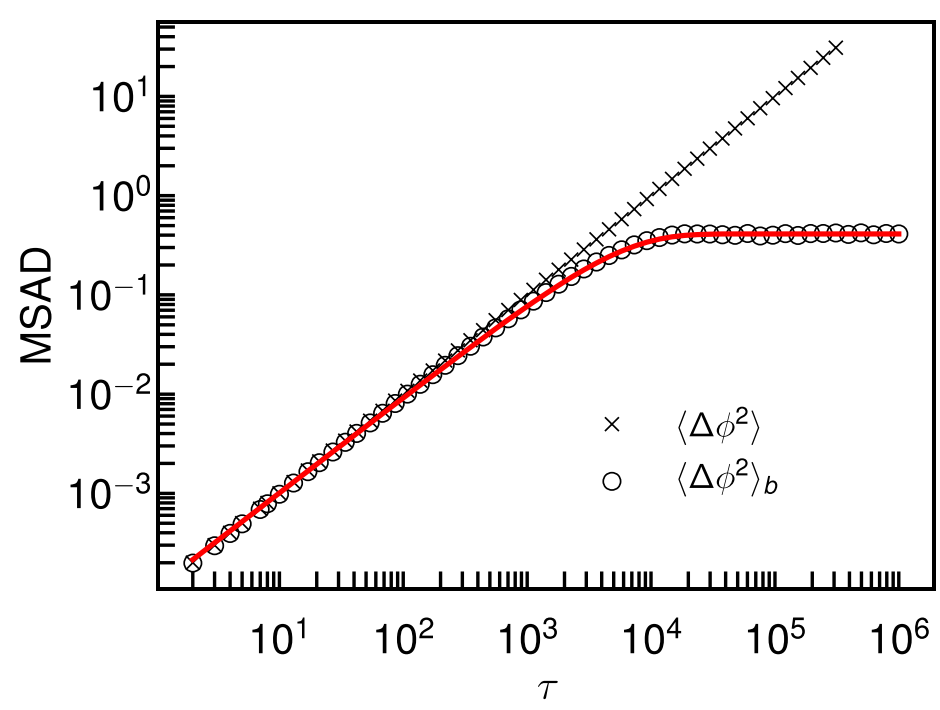

Figure S1. Simulated unbounded 1-d random walk and bounded random walk between 0 and $\pi / 4$. Crosses: Unbounded MSAD. Circles: Bounded MSAD. At short lag time the bounded MSAD is linear and overlaps with the unbounded MSAD. Red curve: fit to Eq. S1.

To determine the stretched exponential parameters, we generate a 1-d random diffusion walk bounded between 0 and $\pi / 2$, and calculate its MSAD, Fig. S1. We then fit Eq. S1 to the MSD with $\xi$ and $\zeta$ as fitting parameters. 


\section{SI2. Example trajectories of interfacial visitors}

Interfacial visitors swimming near the interface show complex swimming paths.
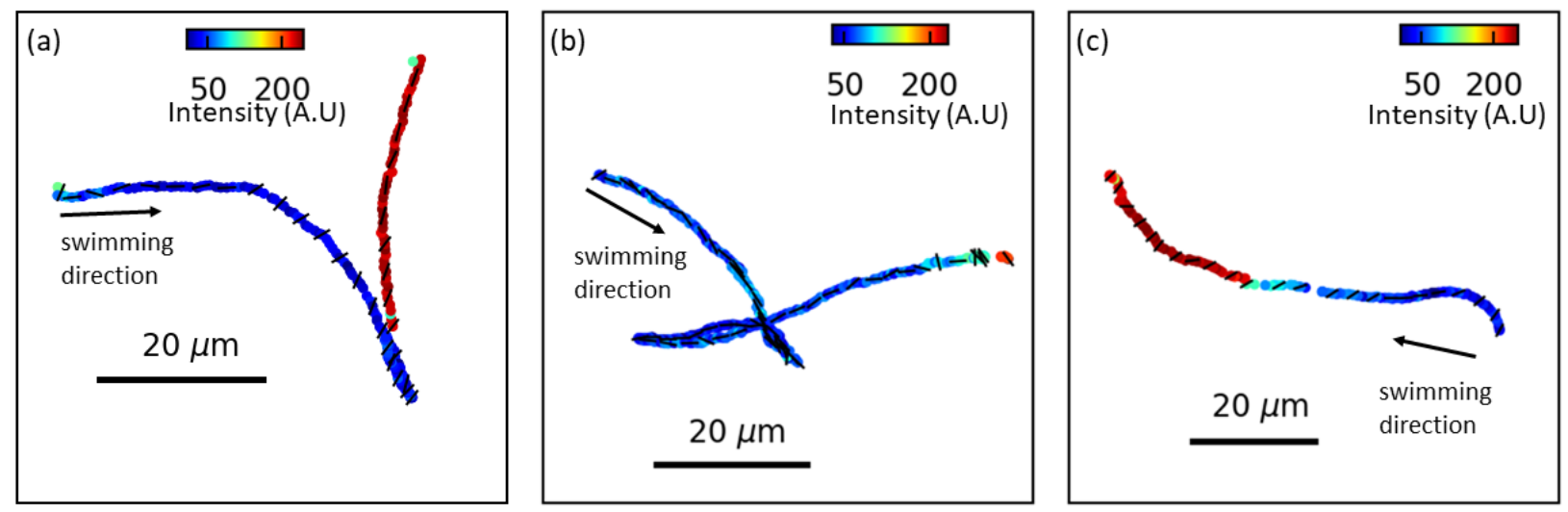

Figure S2: Representative trajectories of interfacial visitors colored by normalized pixel intensity of the bacteria along their path. Brighter segments indicate that bacteria are further from the interface. (a) Bacterium leaves the interfacial region after switching. (b) Bacterium stays in a plane near the interface while switching between CCW and CW motion. (c) Bacterium leaves the interfacial region without a switching event. The black lines indicate the orientation of the visitors' cell bodies. 
SI3. Motility characteristics of the bacterium with curly path
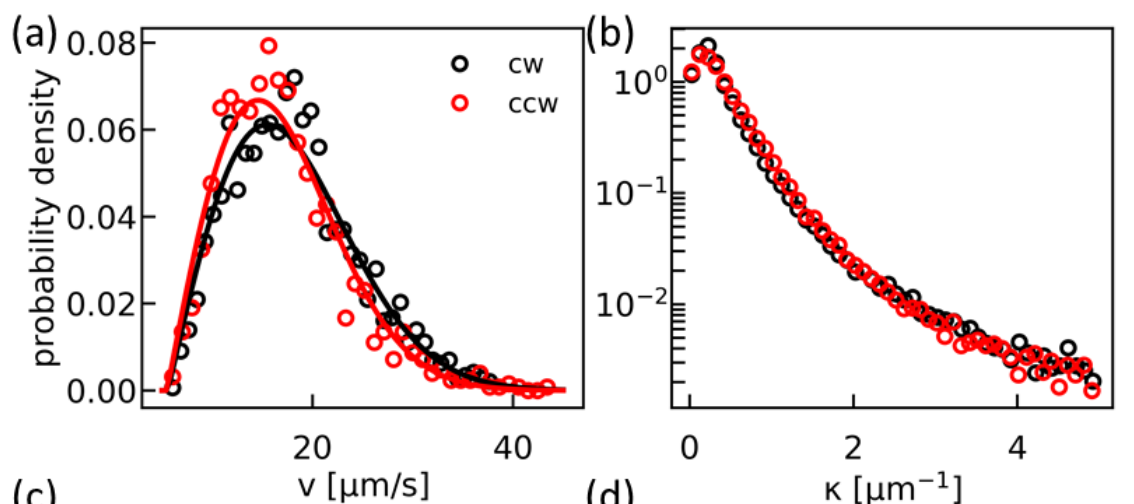

(c)

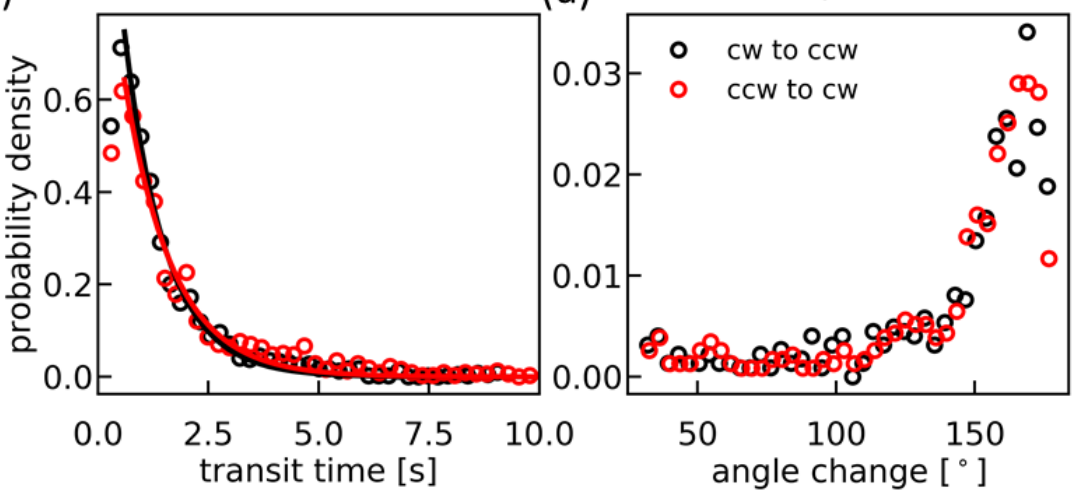

Figure S3: Swimming characteristics of all bacteria with curly paths for CW and CCW paths. (a) Distribution of swimming velocities with Rayleigh fit; (b) curvature of paths; (c) transit time with exponential fits; (d) Angle characterizing change in swimming direction when bacteria switch from CW to CCW and vice versa. 


\section{SI4. The directional autocorrelation function of curly path}

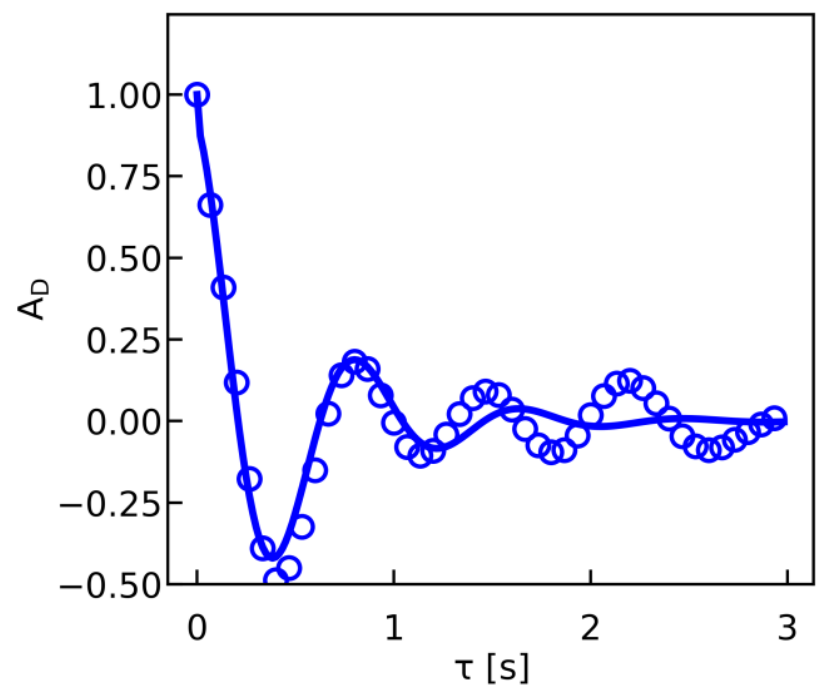

Figure S4: The direction autocorrelation function $\left(A_{D}\right)$ of entire curly trajectory. $A_{D}$ is fitted using Eqn. (4) with fitting parameter $\tau_{c}=0.518 \mathrm{~s}, \tau_{\text {dif }}=5.32 \times 10^{-4} \mathrm{~s}$ and $\tau_{\text {osc }}=0.814 \mathrm{~s}$.

\section{SI5. The MSD of the curvilinear paths}

As discussed in the main text, the MSD of a colloid following a curvilinear path with no thermal diffusion is described by the relationship

$$
\left\langle\Delta r^{2}(\tau)\right\rangle=4 D_{T} \tau+\frac{2 \bar{v}^{2} \tau_{c}}{1+c^{2}} \tau+\frac{2 \bar{v}^{2} \tau_{c}^{2}}{\left(1+c^{2}\right)^{2}}\left\{\left(1-c^{2}\right)\left[e^{-\tau / \tau_{c}} \cos \left(\frac{2 \pi \tau}{\tau_{\mathrm{osc}}}\right)-1\right]-2 c e^{-\tau / \tau_{c}} \sin \left(\frac{2 \pi \tau}{\tau_{\mathrm{osc}}}\right)\right\} \quad \text { Eq. S3 }
$$

where $c=2 \pi \tau_{c} / \tau_{\text {osc }}$. This equation is the general form for a colloid moving in a curvilinear path with average velocity of $\bar{v}$ and oscillation time scale of $\tau_{\text {osc }}$ for which the center of the curly path randomly diffuses with decorrelation time scales of $\tau_{c}$.

We examine Eq. S3 in two limiting cases. First, we consider for a colloid with a persistence path showing a purely ballistic motion with randomizing events at time scale of $\tau_{c}$ and no oscillatory motion. In this limit, $1 / \tau_{\text {osc }}=c=0$, and Eq. S3 reduces to $\left\langle\Delta r^{2}(\tau)\right\rangle=2 \bar{v}^{2} \tau_{c} \tau+2 \bar{v}^{2} \tau_{c}^{2}\left(e^{-\tau / \tau_{c}}-1\right)$. This equation is equivalent to the MSD of persistent trajectories $\left\langle\Delta r^{2}(\tau)\right\rangle=4 D\left[\tau+\tau_{c}\left(e^{-\tau / \tau_{c}}-1\right)\right]$, which is derived in [2] where $D=\bar{v}^{2} \tau_{c} / 2$. Second, if a colloid follows a perfect circle with no diffusion of its center, then $1 / \tau_{c}=1 / c=0$, and we have 


$$
\begin{aligned}
\left\langle\Delta r^{2}(\tau)\right\rangle & =\frac{\bar{v}^{2} \tau_{\mathrm{osc}}^{2}}{2 \pi^{2}\left(1+1 / c^{2}\right)^{2}}\left\{\left(\frac{1}{c^{2}}-1\right)\left[\cos \left(\frac{2 \pi \tau}{\tau_{\mathrm{osc}}}\right)-1\right]-\frac{2}{c} e^{-\tau / \tau_{\mathrm{c}}} \sin \left(\frac{2 \pi \tau}{\tau_{\mathrm{osc}}}\right)\right\} \\
& =\frac{\bar{v}^{2} \tau_{\mathrm{osc}}^{2}}{2 \pi^{2}}\left[1-\cos \left(\frac{2 \pi \tau}{\tau_{\mathrm{osc}}}\right)\right]=\left[\frac{\bar{v} \tau_{\mathrm{osc}}}{\pi} \sin \left(\frac{\pi \tau}{\tau_{\mathrm{osc}}}\right)\right]^{2}
\end{aligned}
$$

The final result of the above equation is the same as equation we found for a perfect curly path in Ref. [2].

\section{SI6. Rotational Autocorrelation Function}

The instantaneous orientation angle of a curly path can be expressed as $\phi(t)=\theta(t)+\varphi(t)$, where $\varphi(t)$ indicates the static noise that fluctuates around $\theta(t)$. Here, $\varphi(t)$ is a stationary random process with zero mean, $\langle\varphi(t)\rangle=0$, and constant variance, $\left\langle\Delta \varphi^{2}(t)\right\rangle=2 \varepsilon_{\phi}^{2}$. The two angles $\theta(t)$ and $\varphi(t)$ are uncorrelated. Thus, we can write the rotational autocorrelation function for $\Delta \phi$ as

$$
A_{R}(\tau)=\frac{\langle\Delta \phi(t) \Delta \phi(t+\tau)\rangle_{t}}{\left\langle\Delta \phi^{2}(t)\right\rangle}=\frac{\langle\Delta \theta(t) \Delta \theta(t+\tau)\rangle_{t}+\langle\Delta \varphi(t) \Delta \varphi(t+\tau)\rangle_{t}}{\left\langle\Delta \theta^{2}(t)\right\rangle+\left\langle\Delta \varphi^{2}(t)\right\rangle}
$$

The angular velocity of the curly motion decorrelates with a characteristic time $\tau_{R}$. The autocorrelation function for $\Delta \theta(t)$ is $\langle\Delta \theta(t) \Delta \theta(t+\tau)\rangle_{t}=\Omega_{0}^{2} \Delta t^{2} \exp \left(-\tau / \tau_{R}\right)$, where $\Omega=d \theta / d t$ is the angular velocity of the bacteria and $\Delta t$ is the time interval between two frames $(\Delta t \approx 0.016 \mathrm{~s}$ in our experiments).

Qualitatively, $2 \varepsilon_{\phi}^{2} \sim 0.25$ (estimated from sub-diffusive plateau of MSAD at short lag time), $\Omega_{0}^{2} \Delta t^{2} \sim 0.01$, so $\left\langle\Delta \phi^{2}(t)\right\rangle$ is dominated by $\left\langle\Delta \varphi^{2}(t)\right\rangle$ term. The term $\langle\Delta \phi(t) \Delta \phi(t+\tau)\rangle_{t}$ is dominated by $\langle\Delta \theta(t) \Delta \theta(t+\tau)\rangle_{t}$ with a value on the order of 0.01 due to the much faster decorrelation of $\langle\Delta \varphi(t) \Delta \varphi(t+\tau)\rangle_{t}$. Thus, the autocorrelation function for $\Delta \phi$ with $\Delta t \approx 0.016 \mathrm{~s}$ shows an uncorrelated behavior due to the high-frequency measurement noises. However, if we calculate $\Delta \phi$ based on a larger time interval $\Delta t$, decorrelation of active rotational motion will dominate with large decorrelation time. We observed an exponential decay with large decorrelation time calculated using larger $\Delta t$. The dependence of shape of $A_{R}$ on the time interval $\Delta t$ is shown in Fig. S5. $A_{R}$ remains roughly unchanged with $\Delta t$ larger than $0.33 \mathrm{~s}$. 


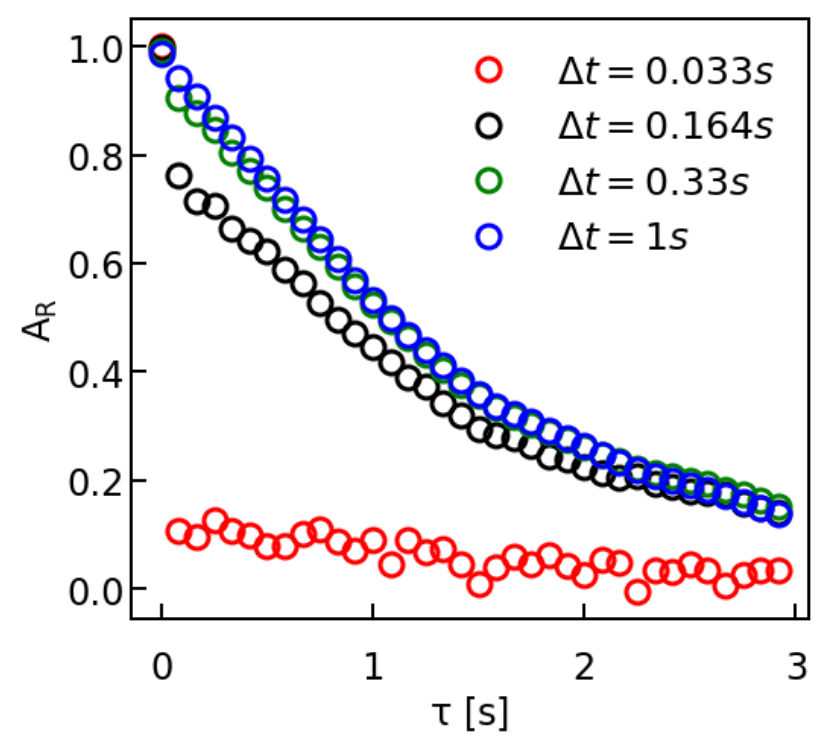

Figure S5: Rotational autocorrelation function $A_{R}$ calculated using different time intervals $\Delta t$ to determine $\Delta \phi$.

\section{SI7. Comparison between PA01 Wild Type (WT) and PA01 $\triangle$ pilC}
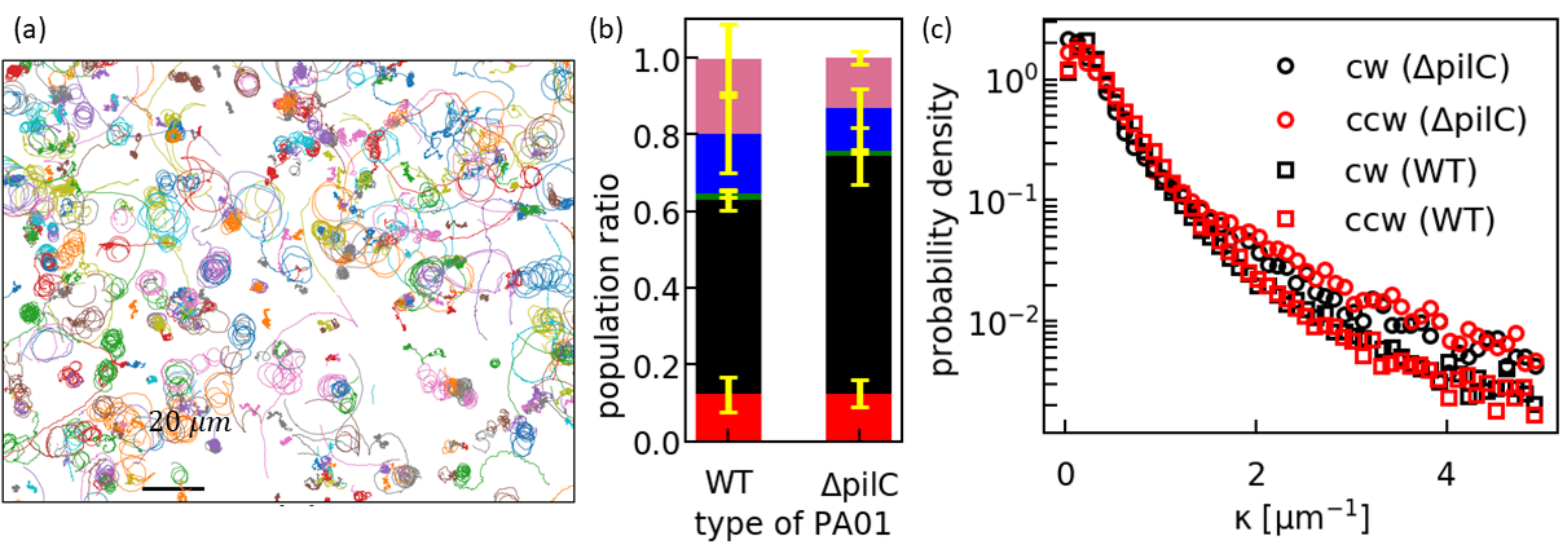

Figure S6: (a) Individual bacterial trajectories of PA01 $\Delta$ pilC strain; only 20 s timespan of trajectories are shown. (b) Population ratio of each trajectory type of depilated PA01 bacteria is roughly the same as wild type bacteria. Colors for each trajectory types match the color in Fig. $2 \mathrm{~g}$ with pink as clustered bacteria, blue as diffusive bacteria, green as pirouette motion, black as curly motion and red as interfacial visitors. The error bar is calculated from three different experiments. (c) Probability distribution of swimming curvature of two bacterial strains. 


\section{SI8. Description of supplemental videos}

The videos enumerated below show the motion of Pseudomonas Aeruginosa PA01 at or near hexadecane-water interface. The videos are observed from water side. (The original videos are flipped vertically)

SI_V1. Interfacial visitor bacterium I: bacterium changes its distance from the interface. The change in the distance is revealed by the change in the pixel intensity of the image of the cell body. As the bacterium approaches the interface, the curvature of its path switches from CCW to CW.

SI_V2. Interfacial visitor bacterium II: bacterium in an unadhered state remains near the interface in both CW and CCW motion.

SI_V3. Brownian diffusive bacterium: video of an immotile bacterium that moves via Brownian motion.

SI_V4. Pirouette motion: video of bacterium with a pirouette motion.

SI_V5. Curly path: video of bacterium swimming in a curvilinear path. The bacterium swims along a CW path in pusher (run) mode and in CCW path in puller (reverse) mode.

\section{References}

[1] Molaei, M.; Atefi, E.; Crocker, J. C. Nanoscale Rheology and Anisotropic Diffusion Using Single Gold Nanorod Probes. Physical Review Letters 2018, 120, 118002.

[2] Vaccari, L.; Molaei, M.; L. Leheny, R.; J. Stebe, K. Cargo carrying bacteria at interfaces. Soft Matter 2018, 14, 5643-5653. 